\title{
Sensorless Control of PMSM by a Four-Switch Inverter with Compensation of Voltage Distortion and Adjustment of Position Estimation Gain
}

\author{
Byeong-Han Kim* and Dong-Myung Lee ${ }^{\dagger}$
}

\begin{abstract}
This paper proposes performance improvement schemes for sensorless PMSM control drive using a four-switch three-phase inverter (so-called B4 inverter). In the proposed scheme, the back-EMF estimation-based sensorless control algorithm is used to control the brushless PMSM without position sensors. In order to have stable operation, this paper presents a gain adjustment scheme that compensates the reduction of stable sensorless operation range as long as the rotor speed increases. In B4 topology, the center point of dc-link capacitors is connected to 3-phase load, and it is prone to have the load current distortion. Hence, to mitigate this problem, a distortion compensation scheme by modifying voltage commands using measured dc-link potentials is proposed in this paper. The validity of the proposed method is evaluated by simulations and experiments.
\end{abstract}

Keywords: Sensorless control, Four-switch inverter, B4 inverter, Matsui, PMSM

\section{Introduction}

To save energy and to improve control performance, field oriented control of a PMSM (Permanent Magnet Synchronous Motor) drive using a B6-type inverter (employing six power switches) is commonly used. Sensors and power switches are relatively expensive, so that cost-effective driving schemes have been widely researched [1-3]. In order to reduce total number of power switches, the B4 topology that uses only 4 switches instead of six switches (B6 topology) was introduced [1].

B4 inverters have serially connected dc-link capacitors as DC power sources, and the center point of the dc-link capacitors is connected to one phase of three phase loads such as motors. Because of this characteristic, there exists the switching mode in which only one high or low side dclink capacitor is involved in power delivery, which results in the voltage unbalance between the high side and low side capacitors. This voltage difference causes distortions in three phase voltages. In order to solve this problem, various researches have been performed [4-5]. A simple compensation method that adjusts switching times considering the dc-link voltage ripples was proposed in [4]. In a current ripple reduction scheme that mitigates the degradation of control performance due to the voltage difference between dc-link capacitors was presented in [5]. In this paper, to use the B4 topology, the distortion of phase voltages is compensated by adding additional voltage components and using the voltage levels of the split dc-link.

Even the B4 topology has less voltage utilization ratio

$\dagger$ Corresponding Author: School of Electronic and Electrical Engineering, Hongik University, Seoul, Korea. (dmlee@hongik.ac.kr)

* School of Electronic and Electrical Engineering, Hongik University, Seoul, Korea.

Received: March 3, 2016; Accepted: May 25, 2016 than that of B6-type and has voltage unbalance problem, applications of B4 inverter are increasing. Masmoudi etal. dealt with DTC (Direct Torque Control) of a BLDC motor by a B4 inverter [6]. Ito etal. proposed an independent control scheme of two PMSMs by using two B4 inverters [7]. In [8], B4 inverters are used for integrating renewable energy sources into microgrid system. Recently, B4 topology is being studied as a provision for the emergency operation against inverter failure as well [9]. To drive PMSM with cost-effective way, this paper applies B4 topology as well as sensorless scheme to PMSM controller. Sensorless control methods $[2,10]$ are researched widely to have cost reduction, to cope with the application under the environment having the difficulty of using sensors, and/or to provide an alternative for the emergency operation against sensor failures.

Sensorless schemes can be classified into two categories as the signal injection method and the back-EMF detection method. The high frequency signal injection method [1012] can get relatively exact rotor position at the low-speed region including the standstill, but has audible noises and additional energy losses due to the injected high frequency signal. Ha etal. proposed a speed and position estimation scheme based on the characteristics of the high-frequency impedance [10]. In [11], for sensorless control, highfrequency pulsating carrier signals were injected into the stationary reference frame. Chen etal. presented a sensorless control scheme for wound rotor synchronous machines by applying high frequency signals into stator windings [12].

On the other hand, the back-EMF based sensorless method $[2,13]$ estimates the rotor position by using stator voltages and currents. The back-EMF based scheme cannot get reliable rotor position in the low-speed region because of relatively small back-EMF in that region. 
Nevertheless, this scheme can be successfully applied to many applications such as compressors that operate at a relatively high speed and have a simple starting-up process $[14,15]$. This paper focuses on the back-EMF based scheme for sensorless position detection because for using the scheme in consumer electronics. The sensorless control schemes with a B4 inverter has been reported in several papers [3, 16-17]. The schemes in [3, 16] are for twophase conduction method, and the method presented in [17] by a Kalman filter is relatively complex. Hence, this paper presents a simple sensorless scheme implemented with a B4 inverter, which is based on the back-EMF detection method reported in Matsui's papers [2,13]. Where, in the sensorless schemes of $[2,13]$ for home appliances application, B6 inverters are involved for power conversion.

The sensorless scheme utilizing Matsui algorithm with a B4 inverter has not been reported. In order to apply the sensorless scheme utilizing Matsui algorithm to a B4 inverter, this paper proposes performance improvement schemes. One is compensation scheme of the distortions in generated pole voltage commands. The other is achieved by adjusting the position estimation gain corresponding to the machine speed. Each algorithm will be explained in Sections 2.3 and 3.3. The validity of the proposed scheme is verified by simulation and experimental results.

\section{Configuration and Operational Principle of the B4 Inverter}

\subsection{Pole voltage commands in B4 topology}

B4 inverters have four switches as shown in Fig. 1. One phase of the load is connected to the center point of their DC power source $[1,4-5]$. On the other hand, conventional B6 inverters (two-level inverters) have six switches without split dc-link.

In this paper, it is assumed that $c$-phase of the load is connected to the center point of the dc-link as displayed in Fig. 1. As the result, $v_{c}$ has zero potential with respect to $c$. It is an equivalent case that addition of $-v_{c s}$ that is opposite direction in phase and equals in amplitude to $v_{c s}$ in the phase voltages. Because the phase voltages even

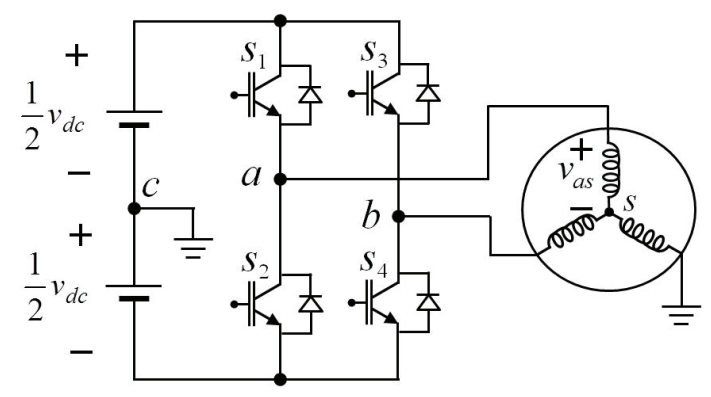

Fig. 1. Configuration of a B4 inverter

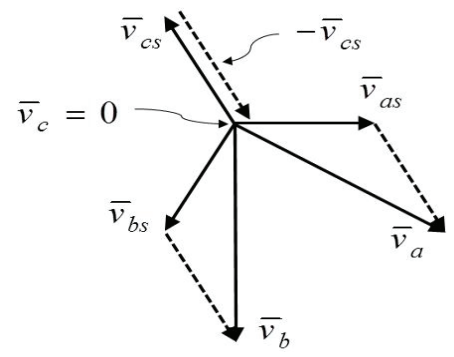

Fig. 2. Phasor diagram of three phase and pole voltages generated by a B4 inverter

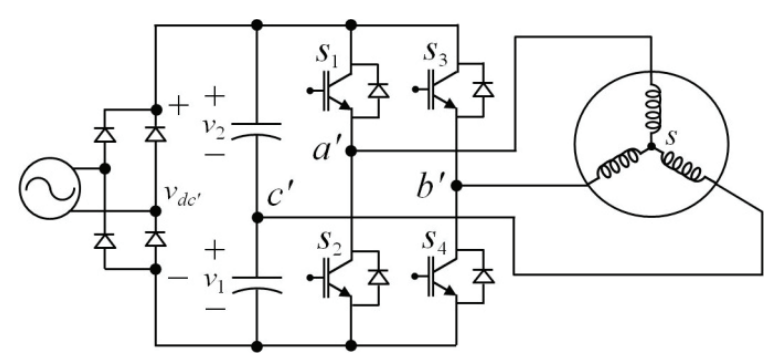

Fig. 3. Motor drive system using a B4 inverter including a rectifier circuit

after adding $-v_{c s}$ have the balanced three-phase voltages such as $v_{a s}, \hat{B}_{b s}$ and $v_{c s}$ illustrated in Fig. 2, B4 inverters can generate the same phase voltages as B6 topology. The pole voltage commands by B4 inverter can be expressed as (1).

$$
v_{a}=v_{a s}-v_{c s}, \quad v_{b}=v_{b s}-v_{c s}, \quad v_{c}=0
$$

\subsection{Voltage fluctuation in the center point of dc-link of B4 inverter}

Fig. 3 shows an actual implementation of B4 inverter, in which a diode-bridge is used for $\mathrm{AC}$ to $\mathrm{DC}$ conversion. In the case of rectifying from $\mathrm{AC}$ to $\mathrm{DC}$, dc-link voltage $\left(v_{d c^{\prime}}\right)$ has voltage ripples due to the influence of the rectifier. In other words, the center point voltage level of dc-link is not constant, which varies according to the load condition. Hence, load currents may be distorted because $v_{a^{\prime}}$ and $v_{b^{\prime}}$ differ from $v_{a}$ and $v_{b}$ due to the split dclink with rectification by a diode-bridge.

$v_{1}$ is not equal to $v_{2}$ with the finite dc-link capacitance, i.e., $v_{1} \neq \frac{1}{2} v_{d c^{\prime}}$, and the potential at the center point of the dc-link can be expressed as (2).

$$
v_{c^{\prime}}=v_{1}-\frac{1}{2} v_{d c^{\prime}}
$$

where, $v_{1}$ is the voltage level of the low-side dc-link capacitor and $v_{d c}$ denotes total dc-link potential, i.e., $v_{d c^{\prime}}=v_{1}+v_{2}$. 


\subsection{Proposed compensation scheme of distortions in phase voltages}

In this paper, to apply sensorless algorithm into the B4 topology, the distortion in phase voltages is compensated by using the information of $v_{1}$ and $v_{d c^{\prime}}$. The proposed scheme is similar to that in [5]. Difference is that this paper does not consider the ideal $v_{d c}$ reference executed in [5] to reduce the complexity in the implementation. The proposed compensation scheme is explained as below.

If $v_{a^{\prime}}$ is commanded as (3), the line to line voltage $\left(v_{a^{\prime}}-v_{c^{\prime}}\right)$ shown in Fig. 3 is equal to the line to line voltage such as $v_{a}-v_{c}=v_{a}$ due to $v_{c}=0$. Hence, the phase voltages shown in Fig. 1 and Fig. 3 are the same each other.

$$
v_{a}=v_{a s}-v_{c s}=v_{a^{\prime}}-v_{c^{\prime}}
$$

With manipulation of (3) for $v_{a^{\prime}}$ and application of (2), modified $a$-pole voltage can be expressed as (4). It shows that $a$-pole voltage $\left(v_{a^{\prime}}\right)$ has the term that reflects the variation in $v_{c^{\prime}}$.

$$
v_{a^{\prime}}=v_{a s}-v_{c s}+v_{c^{\prime}}=v_{a s}-v_{c s}+v_{1}-\frac{1}{2} v_{d c^{\prime}}
$$

Using the same way, the modified $v_{b^{\prime}}$ can be obtained as (5).

$$
v_{b^{\prime}}=v_{b s}-v_{c s}+v_{1}-\frac{1}{2} v_{d c^{\prime}}
$$

On the other hand, because $v_{d c^{\prime}}=v_{1}+v_{2}$, (4) and (5) can be rewritten as (6) and (7). It should be noted that instead of using (6) and (7), (4) and (5) are employed in simulation studies and experiments, because $v_{1}$ and $v_{d c}$ have the common potential with respect to the ground of the digital signal processor (DSP).

$$
\begin{gathered}
v_{a^{\prime}}=v_{a s}-v_{c s}+\frac{1}{2}\left(v_{1}-v_{2}\right) \\
v_{b^{\prime}}=v_{b s}-v_{c s}+\frac{1}{2}\left(v_{1}-v_{2}\right)
\end{gathered}
$$

\section{Sensorless Control Algorithm}

\subsection{Sensorless control scheme based on back-EMF detection}

Fig. 4 shows an overall block diagram of sensorless speed control scheme for a PMSM. Where, the subscript M means an estimated one. The reference current $\left(i^{*}\right)$ is calculated from the error input between the reference speed $\left(\dot{\theta}^{*}\right)$ and the estimated speed $\left(\dot{\theta}_{M_{O}}\right)$ by the PI controller. $v$ is acquired from this reference current input by the PI

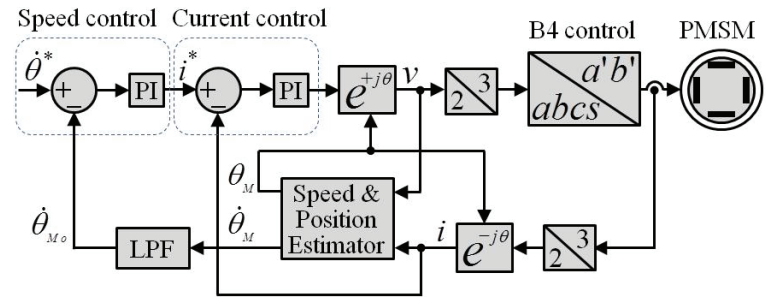

Fig. 4. An overall block diagram for sensorless control of PMSM

controller. $v$ commands are transformed to $v_{a s}, v_{b s}$ and $v_{c s}$ and then pole voltage commands $\left(v_{a^{\prime}}\right.$ and $\left.v_{b^{\prime}}\right)$ are obtained from $v_{a s}, v_{b s}$ and $v_{c s}$ by using (4) and (5).

The sensorless scheme estimates the back-EMF magnitude $\left(e_{M}\right)$ and the rotor position $\left(\theta_{M}\right)$ by using the detected motor current $(i)$ and the reference voltage $(v)$. The motor speed estimate $\left(\dot{\theta}_{M}\right)$ is obtained by the estimated back-EMF. In Fig. $4, \dot{\theta}_{M_{O}}$ represents the filtered motor speed of $\dot{\theta}_{M}$ for the speed feedback, which is obtained by low-pass filtering to eliminate high frequency noise [13].

\subsection{Speed and position estimation algorithm and its stability}

In order to help understanding the gain adjustment scheme, speed and position estimation algorithm and stability of the current model-based sensorless control proposed by Matsui $[2,13]$ are explained in this section.

The $d-q$ axis voltage equation of a salient-pole PMSM is given as (8) and the back-EMF can be expressed as (9).

$$
\begin{gathered}
{\left[\begin{array}{l}
v_{d} \\
v_{q}
\end{array}\right]=\left[\begin{array}{cc}
R+\frac{d}{d t} L_{d} & -\dot{\theta} L_{q} \\
\dot{\theta} L_{d} & R+\frac{d}{d t} L_{q}
\end{array}\right]\left[\begin{array}{l}
i_{d} \\
i_{q}
\end{array}\right]+e\left[\begin{array}{l}
0 \\
1
\end{array}\right]} \\
e=K_{E} \dot{\theta}
\end{gathered}
$$

where, $v_{d}, v_{q}: d$ or $q$ axis stator voltage, $R$ : stator resistance, $L_{d}, L_{q}: d$ or $q$ axis inductance, $\dot{\theta}$ : rotor speed, $i_{d}, i_{q}: d$ or $q$ axis stator current, $e$ : back-EMF, and $K_{E}$ : back-EMF constant.

Fig. 5 depicts an analytical model of PMSM with a PM of sinusoidal flux excitation. The direction of $d-q$ axis on Fig. 5 is aligned in the direction of the actual rotor position. On the other hand, $\gamma-\delta$ axis represents the estimated rotor position (axis), and $\Delta \theta$ means the angular error between $d$ $q$ and $\gamma-\delta$ axis. (10) is the stator voltage equation gotten from (8) by the axis transformation done with respect to the $\gamma-\delta$ axis.

$$
\left[\begin{array}{l}
v_{\gamma} \\
v_{\delta}
\end{array}\right]=\left[\begin{array}{cc}
R+\frac{d}{d t} L_{d} & -\dot{\theta}_{M} L_{q} \\
\dot{\theta}_{M} L_{d} & R+\frac{d}{d t} L_{q}
\end{array}\right]\left[\begin{array}{l}
i_{\gamma} \\
i_{\delta}
\end{array}\right]+e\left[\begin{array}{r}
-\sin \Delta \theta \\
\cos \Delta \theta
\end{array}\right]
$$




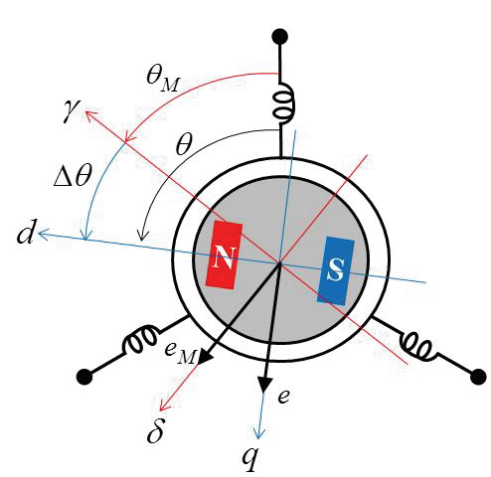

Fig. 5. Analytic model of PMSM

Derivative term of $\gamma-\delta$ axis currents can be expressed as (11). Supposing that the sampling period $(T)$ is shorter enough than the time constant of the stator current, substituting (11) into (10), (12) can be obtained. The superscript $n$ denotes a value at the $n$-th sampling time.

$$
\begin{gathered}
\frac{d}{d t}\left[\begin{array}{l}
i_{\gamma} \\
i_{\delta}
\end{array}\right]=\frac{1}{T}\left(\left[\begin{array}{l}
i_{\gamma}^{n} \\
i_{\delta}^{n}
\end{array}\right]-\left[\begin{array}{l}
i_{\gamma}^{n-1} \\
i_{\delta}^{n-1}
\end{array}\right]\right) \\
{\left[\begin{array}{c}
i_{\gamma}^{n} \\
i_{\delta}^{n}
\end{array}\right]=\left[\begin{array}{cc}
1-\frac{R}{L_{d}} T & \dot{\theta}_{M}^{n-1} \frac{L_{q}}{L_{d}} T \\
-\dot{\theta}_{M}^{n-1} \frac{L_{d}}{L_{q}} T & 1-\frac{R}{L_{q}} T
\end{array}\right]\left[\begin{array}{l}
i_{\gamma}^{n-1} \\
i_{\delta}^{n-1}
\end{array}\right]+\frac{T}{L_{d} L_{q}}\left[\begin{array}{l}
L_{q} v_{\gamma}^{n-1} \\
L_{d} v_{\delta}^{n-1}
\end{array}\right]} \\
+\frac{T}{L_{d} L_{q}} e^{n-1}\left[\begin{array}{c}
L_{q} \sin \Delta \theta^{n-1} \\
-L_{d} \cos \Delta \theta^{n-1}
\end{array}\right]
\end{gathered}
$$

The estimated current $\left(i_{M}^{n}\right)$ calculated by the controller can be rewritten as (13), which is acquired after replacing the actual back-EMF $\left(e^{n-1}\right)$ in the $3^{\text {rd }}$ term of (12) with the estimated back-EMF $\left(e_{M}^{n-1}\right)$ oriented in the direction of $\delta$ axis.

$$
\begin{aligned}
{\left[\begin{array}{l}
i_{M \gamma}^{n} \\
i_{M \delta}^{n}
\end{array}\right]=} & {\left[\begin{array}{cc}
1-\frac{R}{L_{d}} T & \dot{\theta}_{M}^{n-1} \frac{L_{q}}{L_{d}} T \\
-\dot{\theta}_{M}^{n-1} \frac{L_{d}}{L_{q}} T & 1-\frac{R}{L_{q}} T
\end{array}\right]\left[\begin{array}{l}
i_{\gamma}^{n-1} \\
i_{\delta}^{n-1}
\end{array}\right] } \\
& +\frac{T}{L_{d} L_{q}}\left[\begin{array}{l}
L_{q} v_{\gamma}^{n-1} \\
L_{d} v_{\delta}^{n-1}
\end{array}\right]-\frac{T}{L_{q}} e_{M}^{n-1}\left[\begin{array}{l}
0 \\
1
\end{array}\right]
\end{aligned}
$$

The estimated current error $\Delta i\left(=i-i_{M}\right)$ can be represented as (14).

$$
\left[\begin{array}{l}
\Delta i_{\gamma}^{n} \\
\Delta i_{\delta}^{n}
\end{array}\right]=\frac{T}{L_{d} L_{q}}\left[\begin{array}{c}
L_{q} e^{n-1} \sin \Delta \theta^{n-1} \\
L_{d}\left(e_{M}^{n-1}-e^{n-1} \cos \Delta \theta^{n-1}\right)
\end{array}\right]
$$

If $\Delta \theta^{n-1}$ is near zero value, (14) can be approximated as (15).

$$
\left[\begin{array}{l}
\Delta i_{\gamma}^{n} \\
\Delta i_{\delta}^{n}
\end{array}\right] \cong \frac{T}{L_{d} L_{q}}\left[\begin{array}{c}
L_{q} e^{n-1} \Delta \theta^{n-1} \\
-L_{d} \Delta e^{n-1}
\end{array}\right]
$$

Where, the estimated back-EMF error $\left(\Delta e^{n-1}\right)$ is defined as (16).

$$
\Delta e^{n-1}=e^{n-1}-e_{M}^{n-1}
$$

From (15), it can be known that the estimated back-EMF error $\left(\Delta e^{n-1}\right)$ and the estimated position error $\left(\Delta \theta^{n-1}\right)$ are related to $\Delta i_{\delta}^{n}$ and $\Delta i_{\gamma}^{n}$, respectively. Therefore, as shown in (17) and (18), the back-EMF and the rotor position at the $n$-th sampling time can be estimated by multiplying specific gain $K_{e}$ and $K_{\theta}$ to $\Delta i_{\delta}^{n}$ and $\Delta i_{\gamma}^{n}$, respectively. Where, $K_{e}$ and $K_{\theta}$ mean the gain of back-EMF estimation and that of rotor position estimation, respectively.

$$
\begin{aligned}
& e_{M}^{n}=e_{M}^{n-1}-K_{e} \Delta i_{\delta}^{n} \\
& \theta_{M}^{n}=\theta_{M}^{n-1}+\frac{T}{K_{E}} e_{M}^{n}+K_{\theta} \operatorname{sgn}\left(\dot{\theta}_{M_{o}}^{n-1}\right) \Delta i_{\gamma}^{n} \\
& \operatorname{sgn}\left(\dot{\theta}_{M_{o}}^{n-1}\right)=\left\{\begin{array}{c}
1: \dot{\theta}_{M_{o}}^{n-1} \geq 0 \\
-1: \dot{\theta}_{M_{o}}^{n-1}<0
\end{array}\right.
\end{aligned}
$$

Where, the second term in the right side of (18) is the amount of position change during one sampling time. $\dot{\theta}_{M}^{n}$ is the estimated speed, which has the relationship such as $\left(\theta_{M}^{n}-\theta_{M}^{n-1}\right) / T$. Hence by utilizing (18), the rotor speed can be obtained as (20).

$$
\dot{\theta}_{M}^{n}=\frac{\theta_{M}^{n}-\theta_{M}^{n-1}}{T}=\frac{e_{M}^{n}}{K_{E}}+\frac{K_{\theta}}{T} \operatorname{sgn}\left(\dot{\theta}_{M_{o}}^{n-1}\right) \Delta i_{\gamma}^{n}
$$

Because the estimated speed $\left(\dot{\theta}_{M}^{n}\right)$ may include detection noise, instead of using $\dot{\theta}_{M}^{n}, \dot{\theta}_{M_{o}}^{n}$, which is filtered one by using (21), is employed.

$$
\left.\begin{array}{l}
\dot{\theta}_{M_{o}}^{n}=\frac{e_{M}^{n}}{K_{E}}+\Delta \dot{\theta}_{M_{o}}^{n} \\
\Delta \dot{\theta}_{M_{o}}^{n}=\Delta \dot{\theta}_{M_{o}}^{n-1}+K\left(\Delta \dot{\theta}_{M}^{n}-\Delta \dot{\theta}_{M_{o}}^{n-1}\right)
\end{array}\right\}
$$

where, $K(0<K<1)$ is the smoothing factor.

The stability of the estimation algorithm can be mentioned as below. Assuming that the speed response of the motor is slow compared to that of estimation process, the motor speed and the back-EMF during the sampling time can be considered as constant. Hence it allows the position $\left(\theta^{n}\right)$ to be written as (22).

$$
\theta^{n}=\theta^{n-1}+\frac{T}{K_{E}} e^{n-1}
$$

The estimation errors $\left(\Delta e^{n}\right.$ and $\left.\Delta \theta^{n}\right)$ are expressed as (23) by manipulating (17), (18), and (22).

$$
\left[\begin{array}{l}
\Delta e^{n} \\
\Delta \theta^{n}
\end{array}\right]=\left[\begin{array}{cc}
1-K_{e} \frac{T}{L_{q}} & 0 \\
\frac{T}{K_{E}}\left(1-K_{e} \frac{T}{L_{q}}\right) & 1-K_{\theta}\left|e^{n-1}\right| \frac{T}{L_{d}}
\end{array}\right] *\left[\begin{array}{c}
\Delta e^{n-1} \\
\Delta \theta^{n-1}
\end{array}\right]
$$




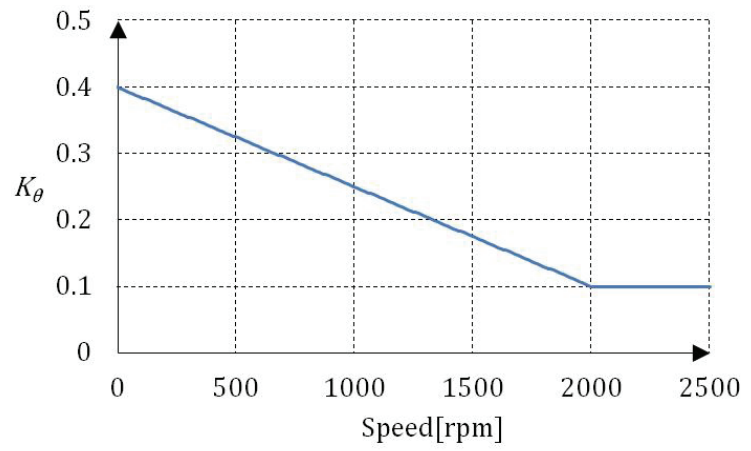

Fig. 6. The proposed variable estimation gain pattern used in experiments, which corresponds to rotor speed

If the absolute values of the eigenvalues of the system matrix in (23), which are $\left|1-K_{e} \frac{T}{L_{q}}\right|$ and $\left|1-K_{\theta}\right| e^{n-1}\left|\frac{T}{L_{d}}\right|$, are less than 1, this estimation algorithm is stable. Therefore, the estimation gains $\left(K_{e}\right.$ and $\left.K_{\theta}\right)$ having the following range give a stable sensorless operation.

$$
0<K_{e}<2 \frac{L_{q}}{T}, 0<K_{\theta}<2 \frac{L_{d}}{\left|e^{n-1}\right| T}
$$

\subsection{Proposed gain adjustment scheme}

(24) shows that $K_{\theta}$ changes with $e^{n-1}$, and $K_{\theta}$ value for stable operation decreases as the speed increases because the back-EMF term $\left(e^{n-1}\right)$ is in the denominator. Hence, reducing $K_{\theta}$ in accordance with the speed increase will be able to compensate the decline in the stable operation range.

Fig. 6 depicts $K_{\theta}$ pattern proposed in this paper. The linearly changing $K_{\theta}$ with a lower limit is presented. Since low $K_{\theta}$ results in a slow conversion and then it causes delays in the estimation process. Hence, $K_{\theta}$ is set to have a lower limit. In contrast, higher limit value of $K_{\theta}$ is determined with the consideration of stable operation under the environment having disturbances such as measurement noises. $K_{\theta}$ pattern was obtained from simulation studies and applied in experiments. In the proposed scheme $K_{e}$ has the constant value of 2 in carrying out the simulations and experiments. As shown in (24), only $L_{q}$ is involved in determining $K_{e}$. In contrast, $K_{\theta}$ range for ensuring a stable operation is affected by the rotor speed. Even considering $L_{q}$ variation according to the degree of the saturation, this range of $K_{e}$ expressed in (24) varies not significantly so that it is possible to obtain a stable sensorless operation with fixed $K_{e}$ value.

\subsection{Mode selection for aligning rotor at start-up}

In this paper, by using a specific gate pattern a rotor is arranged in a predetermined initial position prior to start. The proposed sensorless scheme is devised for the applications such as washers or refrigerators where the
Table 1. Switch modes of the B4 inverter and their corresponding switch status ( 0 or 1 means off or on, respectively)

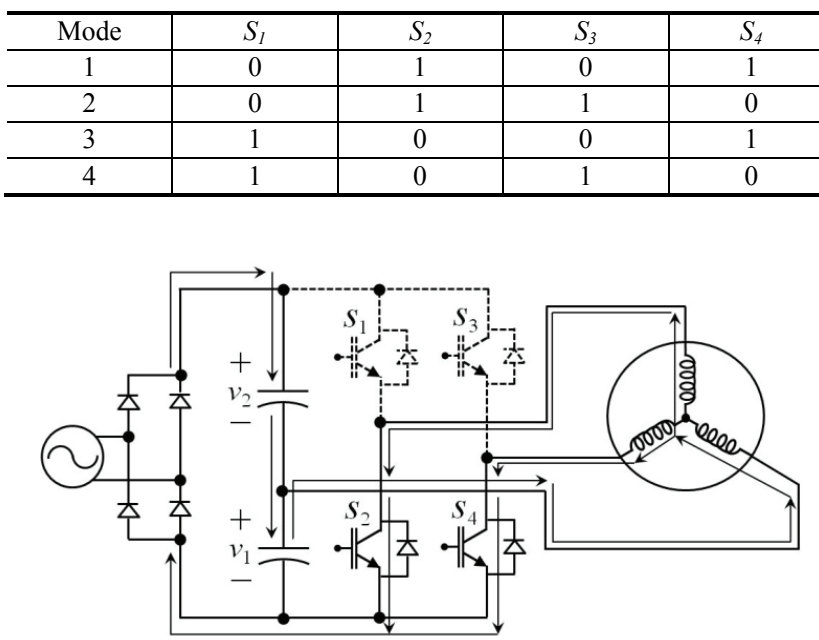

Fig. 7. Power flow in mode 1

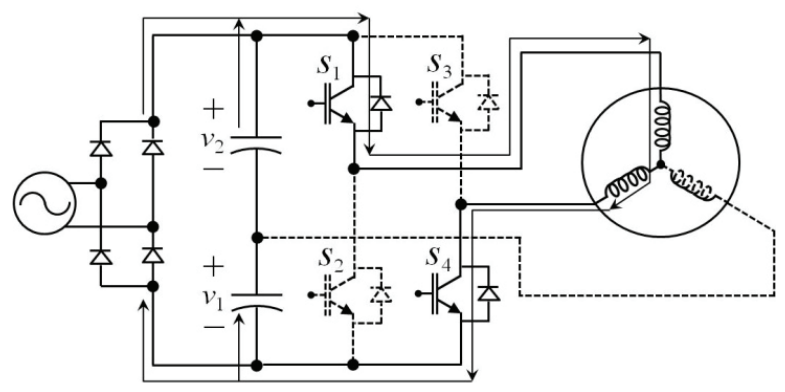

Fig. 8. Power flow in mode 3

initial alignment is allowable, hence the forced alignment has been carried out.

In the case of a B6 inverter, 8 switching modes are possible. In contrast, 4 modes summarized in Table 1 are allowable in B4 inverters. In the case of a B4 inverter, 2 modes of them are selected and used for aligning the rotor. The reason for it is as follows.

Fig. 7 shows the power flow to the load in mode 1. In this mode, as the power is delivered through only the lower-side capacitor having $v_{1}$ potential, the imbalance between $v_{1}$ and $v_{2}$ arises (i.e., making $v_{2}>v_{1}$ ) and is intensified in proportion to the alignment time. Similar phenomenon occurs during the alignment period done by switching mode 4 , which makes $v_{1}>v_{2}$. On the other hand, in mode 2 or 3 , the power is delivered to load side through both dc-link capacitors as shown in Fig. 8. Therefore, the initial alignment has been conducted with the voltage vector of mode 2 or mode 3 in this study.

\section{Simulation Results}

Simulation studies have been done with Matlab/ 
Simulink $^{\circledR}$, and specification of PMSM and control parameter for simulations and experiments are summarized in Table 2. Fig. 9 displays simulation results done with $K_{\theta}=10, K_{\theta}=0.1$, and $K_{\theta}=1$, respectively. In each

Table 2. Specifications for simulation and experiments

\begin{tabular}{c|c}
\hline Parameter & Value \\
\hline Power supply voltage & $220\left[\mathrm{~V}_{\mathrm{rms}}\right]$ \\
\hline Power supply frequency & $60[\mathrm{~Hz}]$ \\
\hline Number of poles & 4 \\
\hline Stator phase resistance & $2.5[\Omega]$ \\
\hline$d$-axis inductance & $96[\mathrm{mH}]$ \\
\hline$q$-axis inductance & $129[\mathrm{mH}]$ \\
\hline PM flux linkage & $0.15[\mathrm{~V} \cdot \mathrm{s}]$ \\
\hline Switching frequency & $16[\mathrm{kHz}]$ \\
\hline dc-link capacitor & $1,000[\mu \mathrm{F}]$ each \\
\hline
\end{tabular}

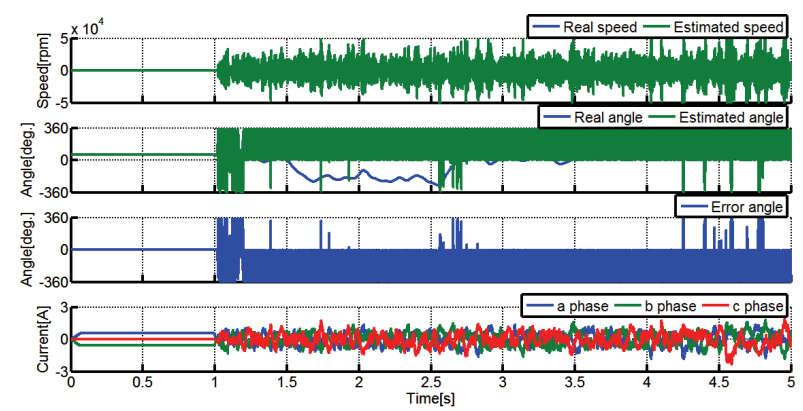

(a)

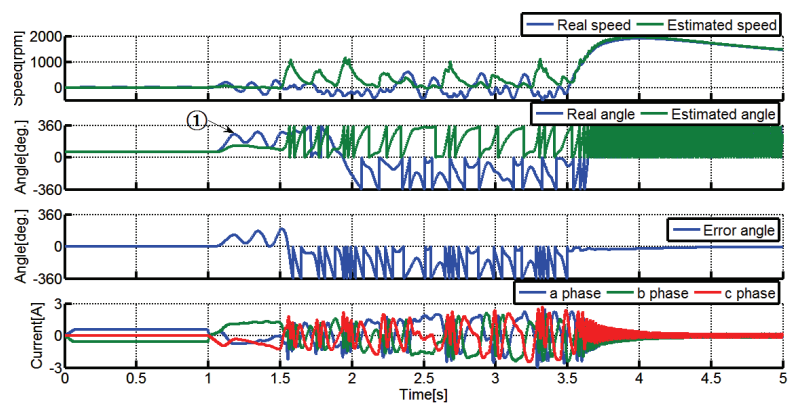

(b)

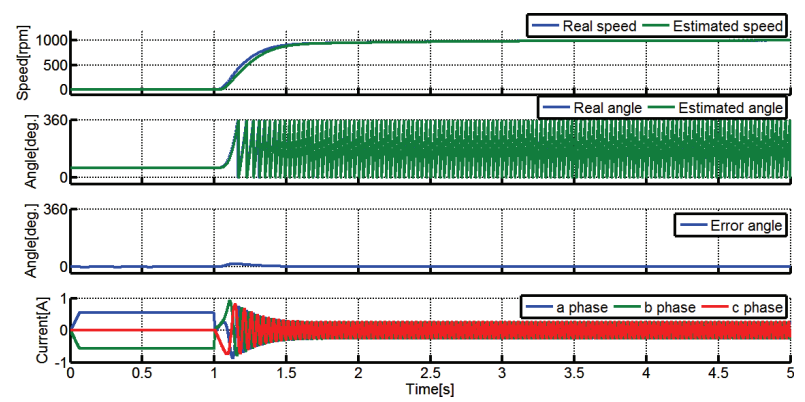

(c)

Fig. 9. Simulation results carried out with different $K_{\theta}$ values (a) $K_{\theta}=10$, (b) $K_{\theta}=0.1$, and (c) $K_{\theta}=1$, respectively. From top to bottom: actual \& estimated rotor speed, actual \& estimated position angle, angle difference between actual \& estimated one, and motor phase currents case, $K_{\theta}$ is not changing one but fixed one while the simulation is in progress. These simulations were performed in order to obtain the proper range of $K_{\theta}$ for experiments. The distortion compensation technique explained previously was applied and the gate pattern of mode 3 was selected for initial alignment process conducted for 1 second.

Fig. 9(a) shows simulation result conducted with $K_{\theta}=10$, and it displays unstable operation that has the angle discrepancy in the actual rotor position and the estimated one. The estimated rotor position includes large noises. Fig. 9(b) displays simulation results carried out with $K_{\theta}=0.1$. It shows that there exists the large angle difference between the actual and estimated one at the initial start-up period (refer to the part pointed by arrow (1)). Although the initial position estimation is incorrect, once estimated position is synchronized to the actual angle (refer to the waveforms around $t=3.5$ seconds), the angle difference goes to zero as the rotor accelerates. Fig. 9(c) illustrates the simulation result with $K_{\theta}=1$ and displays good sensorless control operation. Fig. 9 indicates that large $K_{\theta}$ value like 10 gives unstable operation and incorrect estimation result with lots of noise in all speed range. Meanwhile, lower $K_{\theta}$ like 1 gives stable operation.

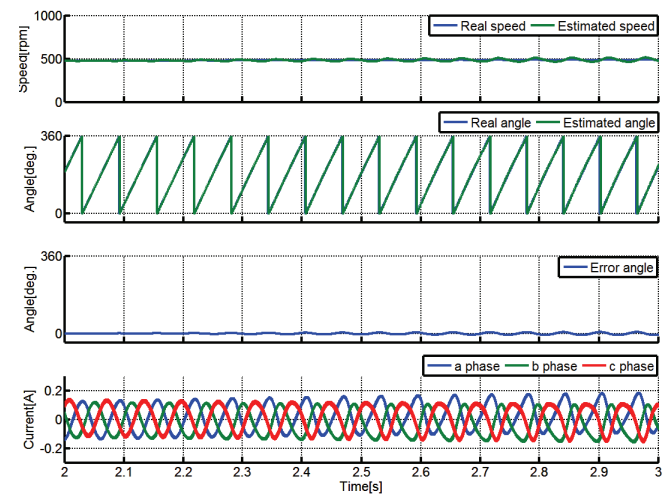

(a)
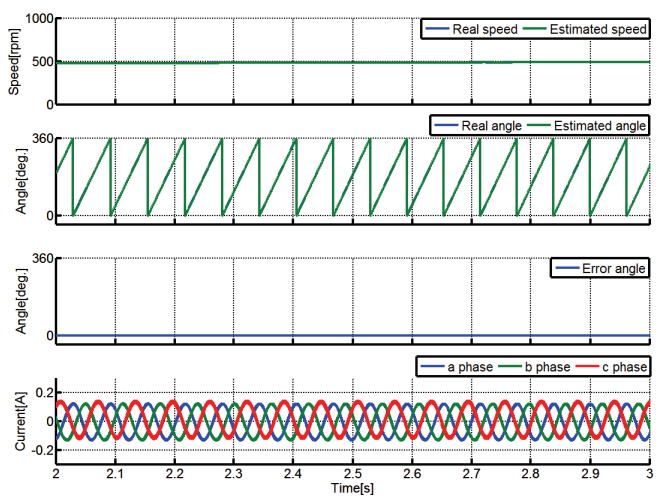

(b)

Fig. 10. Simulation results operated at $500 \mathrm{rpm}$ : (a) without the voltage distortion compensation scheme but starting with the proposed compensation scheme, and (b) with the proposed scheme 
Whereas, the sensorless performance with $K_{\theta}=0.1$ illustrates unstable operation in the initial start-up period but has stable in high-speed region as expected in Section 3.3. With reference to the simulation results, the $K_{\theta}$ less than 0.4 (i.e, $K_{\theta} \leq 0.4$ ) were selected in experiments as displayed in Fig. 6 considering the experimental environment that has disturbances such as measurement noises which is absent in the simulation studies.

Fig. 10 illustrates simulation waveforms operated at 500 rpm (a) without and (b) with the proposed scheme. Fig. 10 clearly shows the current waveforms with the proposed compensation technique have more balanced than those without the distortion compensation algorithm. In case of Fig. 10(b) for without the voltage distortion scheme, the motor can not start without compensating the voltage distortion, so that at the start-up the proposed scheme was applied and then the compensation scheme has been disabled to compare the control performances with and without the distortion mitigation algorithm.

\section{Experimental Verifications}

Fig. 11 is the photograph of experimental setup for sensorless controller of a three-phase PMSM driven by a four-switch inverter. The PMSM and control parameters are the same as that for simulation study. The control algorithm was implemented by the TMS320F28335 DSP. $a$ - and $b$ - phase currents, the dc-link voltage, and the lowside dc-link capacitor voltage were measured. Phase currents were controlled by using two IGBT modules. One IGBT module shown in Fig. 11 includes one high-side IGBT and one low-side IGBT.

Fig. 12 shows experimental results of motor phase currents and actual \& estimated position angles with the proposed distortion compensation technique and $K_{\theta}$ adjustment scheme. The encoder was attached to show the actual position for the verification of the proposed algorithm. The estimated position was displayed by the DA conversion circuit in the experimental setup.

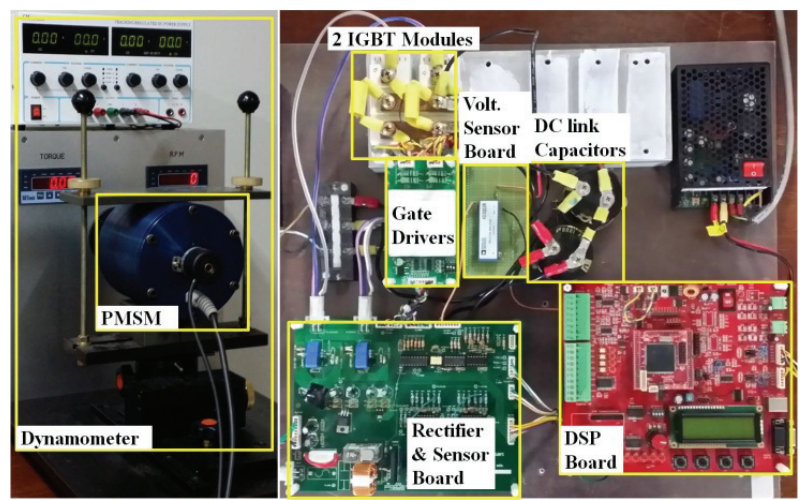

Fig. 11. Photograph of experimental setup for sensorless control of three-phase PMSM using a B4 inverter
Fig. 12(a) and (b) display experimental waveforms of acceleration from zero rpm to $1,000 \mathrm{rpm}$ with initial alignment for 1 second by (a) mode 1 and (b) mode 3, separately. The experimental waveforms of Fig. 12(b) well agree with the simulation waveforms of Fig. 10(c). Fig. 12(b) shows stable acceleration and steady state operation while Fig. 12(a) does not. The reason is as follows. As shown in Fig. 12(a), the voltage reduction in the one of dclink capacitor delivering the power to the load made $a$ - and $b$-phase currents $0 \mathrm{~A}$ (refer to the arrow (1)), so that the B4 inverter lost the ability to control phase currents and it resulted in errors of the aligned position and the unstable start-up process (refer to the arrow (2) for the start-up point after the alignment). Therefore, the forced alignment done for B4 inverters should be executed by mode 2 or 3 as expected in Section 3.4.

Fig. 13(a) and (b) show experimental waveforms under low-speed operation (100 rpm) with (a) fixed $K_{\theta}$ and (b) linearly changing $K_{\theta}$, respectively. $K_{\theta}$ of Fig. 13(a) is 0.1 . Fig. 13(a) depicts that the fixed $K_{\theta}$ value does not has a stable operation. Due to the small $K_{\theta}$, which causes delays in the estimation, there is relatively significant amount of the angle difference between the actual position and the estimated one at the beginning about maximum $\pi$ radians as well as during the steady-state about $\pi / 5$ radians.

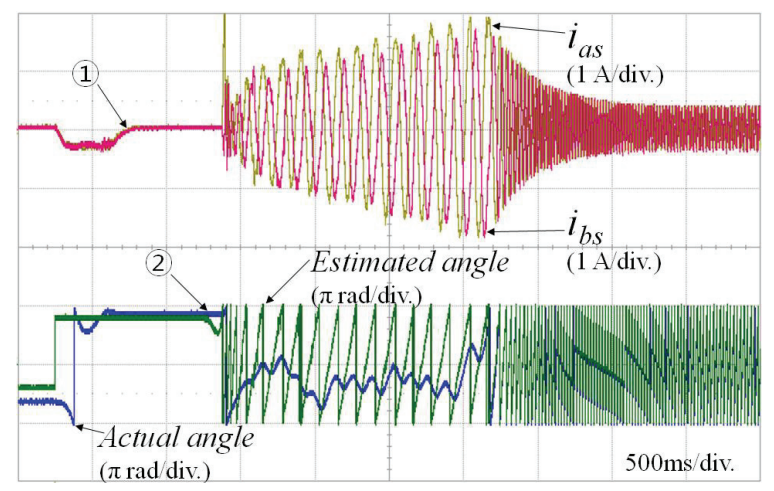

(a)

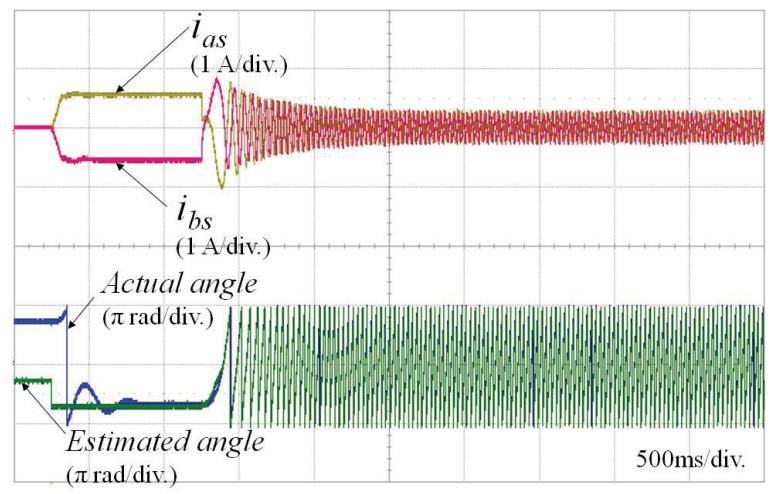

(b)

Fig. 12. Experimental results with the proposed voltage compensation and $K_{\theta}$ adjustment scheme, aligned by (a) mode 1 and (b) mode 3 , respectively 


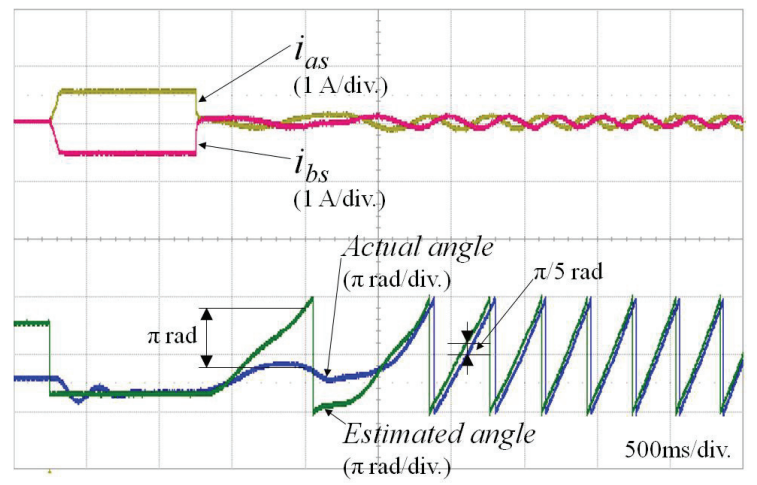

(a)

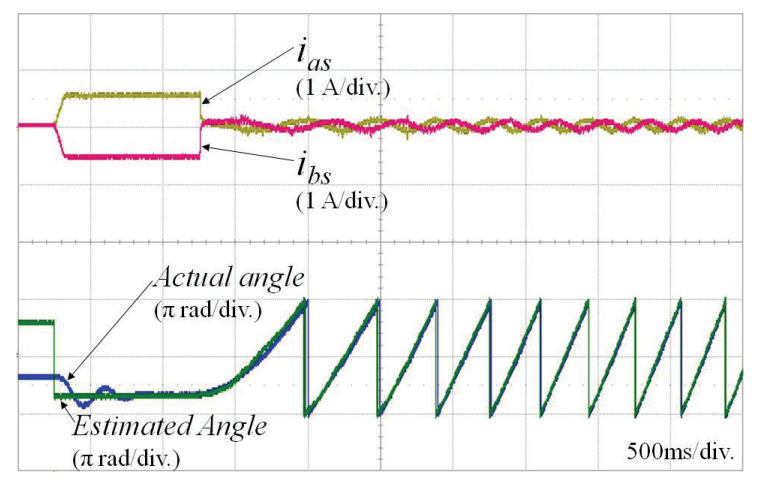

(b)

Fig. 13. Experimental results done under $100 \mathrm{rpm}$ operation: (a) without gain adjustment scheme and (b) with the proposed gain adjustment algorithm

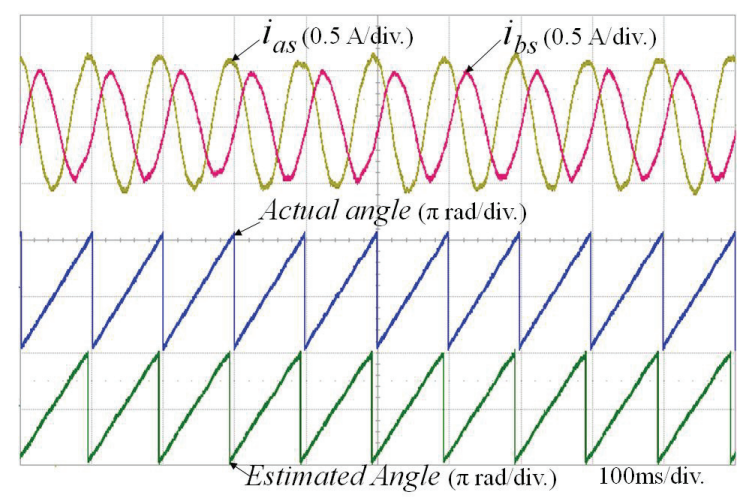

(a)

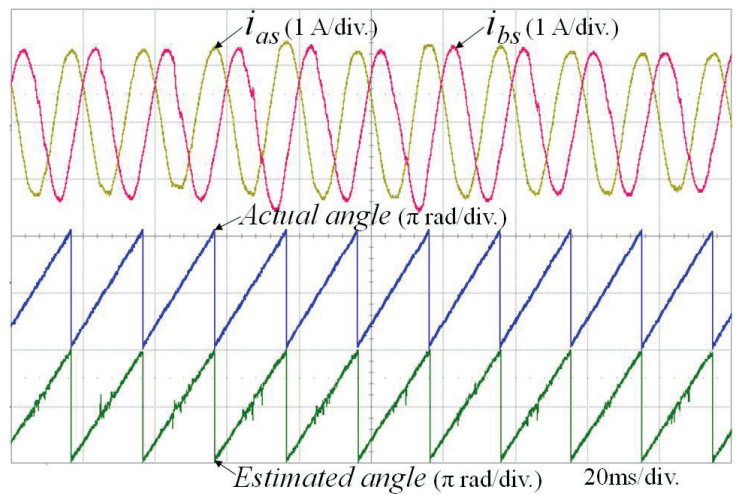

(a)

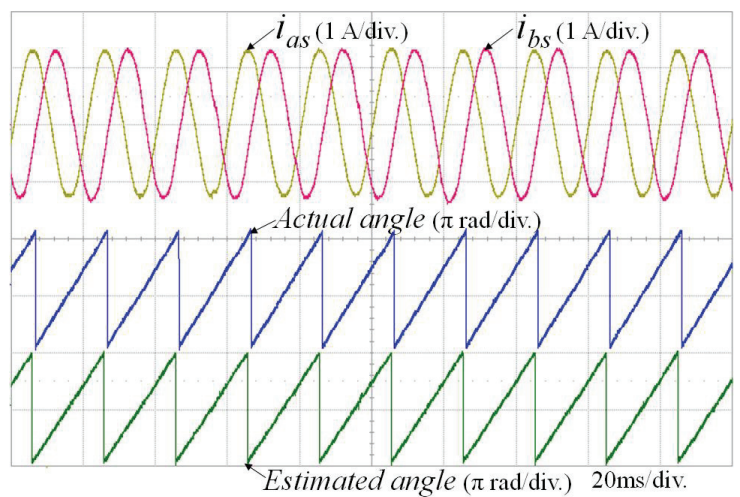

(b)

Fig. 14. Experimental results for 1,500 rpm operation: (a) without gain adjustment scheme and (b) with the proposed gain adjustment scheme

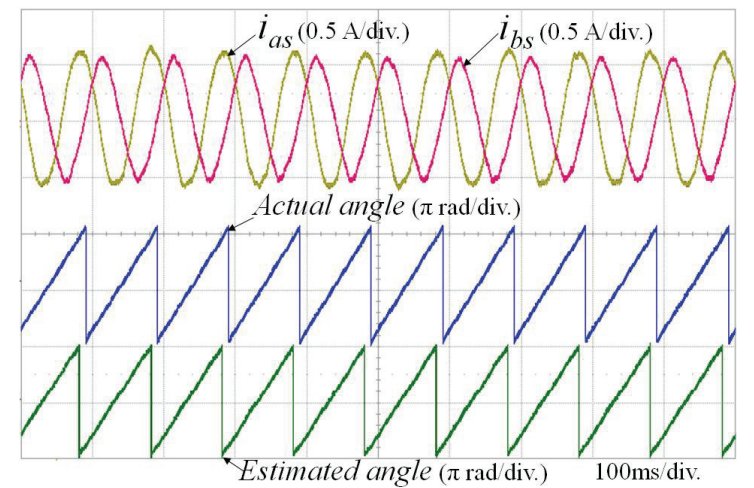

(b)

Fig. 15. Experimental results operated at $300 \mathrm{rpm}$ : (a) without voltage distortion compensation and (b) with the proposed compensation scheme

The angle difference has been reduced after reaching the steady-state. Hence, Fig. 13(a) says that higher estimation gain is needed at the initial starting stage. Fig. 13(b) shows experimental results performed by applying the proposed scheme, variable $K_{\theta}$ in accordance with the motor speed. It can be known that the proposed method accurately estimates the rotor position during the steady state and the transient state as well.
To see the efficacy of variable $K_{\theta}$ in high-speed range, experiments were carried out at 1,500 rpm. Fig. 14 (a) and (b) show experimental results for 1,500 rpm operation with (a) fixed $K_{\theta}(=0.4)$ and (b) variable $K_{\theta}$, respectively.

Fig. 14(a) illustrates that the highly-specified fixed $K_{\theta}$ generates noises in the estimated position signal, and resultant distortion in the motor current. In contrast, Fig. 14(b) depicts that the proposed scheme, in which $K_{\theta}$ is 
proportionally reduced as the speed increases, removes the noise in the estimated speed and generates sinusoidal motor currents.

Fig. 15 shows experimental waveforms conducted under $300 \mathrm{rpm}$ operation (a) without and (b) with the proposed voltage distortion compensation scheme. In both 15 (a) and (b) cases, $K_{\theta}$ varies corresponding to the rotor speed. It should be mentioned that the proposed voltage compensation scheme has been applied in the experiments shown in Figures 12-14. From Fig. 15, it is obvious that the current waveforms with the proposed compensation scheme are much more balanced compared to the current waveforms in the absence of distortion compensation algorithm.

\section{Conclusion}

This paper proposed performance improvement schemes for sensorless PMSM controller using a four-switch threephase inverter. In B4 topology, the center point of dc-link capacitors is connected to 3-phase load, and it is prone to have the load current distortion. The distortion of the motor phase voltages was compensated by modifying the pole voltage commands. In order to ensure stable operation, this paper presented a gain adjustment scheme that compensates the reduction of stable sensorless operation range as the rotor speed increases. The gain pattern has been determined through simulation studies and applied in experiments. The efficacy of the proposed scheme has been verified by simulation and experimental results.

\section{Acknowledgment}

This work was supported by the Korea Institute of Energy Technology Evaluation and Planning (KETEP) granted financial resource from the Ministry of Trade, Industry \& Energy, Republic of Korea(No.20152010103540), and this research was supported by Basic Science Research Program through the National Research Foundation of Korea(NRF) funded by the Ministry of Science, ICT and Future Planning(NRF- 2016R1A2B4011954).

\section{References}

[1] H. van der Broeck and J. van Wyk, "A comparative investigation of a three-phase induction machine with a component minimized voltage-fed inverter under different control options," IEEE Trans. on Indus. Appli., vol. 20, no. 2, pp. 309-320, Mar./Apr. 1984.

[2] N. Matsui, "Sensorless PM brushless DC motor drives," IEEE Trans. Ind. Electron., vol. 43, no. 2, pp. 300-308, Apr. 1996.

[3] C. T. Lin, C. W. Hung, and C. W. Liu, "Position sensorless control for four-switch three-phase brushless DC motor drives," IEEE Trans. Power Electron., vol. 23, no. 1, pp. 438-444, Jan. 2008.

[4] D. M. Lee, J. B. Park, and H. A. Toliyat, "A simple current ripple reduction method for B4 inverters," Journal of Elect \& Tech., vol. 8, no. 5, pp. 1062-1069, 2013.

[5] D. M. Lee, J. W. Jung, S. W. Heo, and T. H. Kim, "Analytic model of four-switch inverter-fed driving system for wye or delta-connected motor with current ripple reduction scheme," Journal of Elect \& Tech., vol. 11, no. 1, pp. 109-116, 2016.

[6] M. Masmoudi, B. Badsi, and A. Masmoudi, "DTC of B4-inverter-fed BLDC motor drives with reduced torque ripple during sector-to-sector commutations," IEEE Trans. Power Electron., vol. 29, no. 9, pp. 4855-4865, Sep. 2014.

[7] S. Ito, T. Moroi, Y. Kubo, K. Matsuse, and K. Rajashekara, "Independent control of two permanent magnet synchronous motors fed by a four-leg inverter," IEEE Trans. Indus. Appli., vol. 51, no. 1, pp. 753-760, Jan./Feb. 2015.

[8] S. Dasgupta, S. N. Mohan, S. K. Sahoo, and S. K. Panda, "Application of four-switch-based three-phase grid-connected inverter to connect renewable energy source to a generalized unbalanced microgrid system," IEEE Trans. Indus. Elect., vol. 60, no. 3, pp. 12041215, March 2013.

[9] W. Zhang, D. Xu, P. N. Enjeti, H. Li, J. T. Hawke, and H. S. Krishnamoorthy, "Survey on fault-tolerant techniques for power electronic converters," IEEE Trans. Power Electron., vol. 29, no. 12, pp. 63196331, Dec. 2014.

[10] J. Ha, K. Ide, T. Sawa, and S. Sul, "Sensorless rotor position estimation of an interior permanent-magnet motor from initial states," IEEE Trans. Ind. Appli., vol. 39, no. 3, pp. 761-767, May/Jun. 2003.

[11] J. M. Liu and Z. Q. Zhu, "Novel sensorless control strategy with injection of high-frequency pulsating carrier signal into stationary reference frame," IEEE Trans. Ind. Appli., vol. 50, no. 4, pp. 2574-2583, July 2014.

[12] Z. Chen, X. Deng, K. Huang, W. Zhen, and L. Wang, "Sensorless control of wound rotor synchronous machines based on high-frequency signal injection into the stator windings," Journal of Power Electr., vol. 13, no. 4, pp. 669-678, July 2013.

[13] T. Takeshita, M. Ichikawa, J. Lee, and N. Matsui, "Back emf estimation-based sensorless salient-pole brushless DC motor drives," Trans. Inst. Elect. Eng. Jpn., vol. 117-D, no. 1, pp. 98-104, 1997.

[14] K. H. Nam, "AC motor control and electric vehicle applications," CRC Press, 2010.

[15] K. W. Lee and J. I. Ha, "Evaluation of back-EMF estimators for sensorless control of permanent magnet synchronous motors," Journal of Power Electr., vol. 
12, no. 4, pp. 1-11, July 2012.

[16] H. Niasar, A. Vahedi, and M. Moghbelli, "A novel position sensorless control of a four-switch brushless DC motor drive without phase shifter," IEEE Trans. Power Electron., vol. 23, no. 6, pp. 3079-3087, Nov. 2008.

[17] J. S. Jang, B. G. Park, T. S. Kim, D. M. Lee, and D. S. Hyun, "Sensorless control of four-switch three-phase PMSM drive using extended Kalman filter," Proc. IEEE IECON, pp. 1368-1372, June 2008.

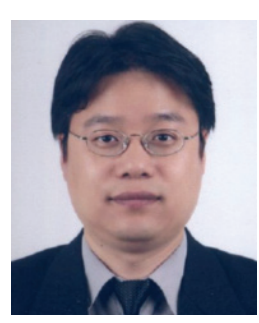

Byeong-Han Kim received his B.S., M.S., and Ph. D. degrees in Electronic and Electrical Engineering from Hongik University, Seoul, Korea, in 1996, 1998, and 2016, respectively. From 1998 to 2011, He worked for Carrier Limited Korea R\&D Center, Seoul, Korea. Since 2011, he was employed by the Samsung Electro-Mechanics Co., Ltd., Suwon, Korea, as a Senior Engineer. His current research interests include three phase PMSM sensorless drive, single phase PMSM high speed drive, and SRM high speed drive.

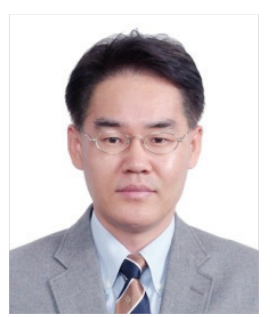

Dong-Myung Lee received his B.S. and M.S. in Electrical Engineering from Hanyang University, Seoul, Korea, in 1994 and 1996, respectively, and his Ph.D. in Electrical and Computer Engineering from the Georgia Institute of Technology, Atlanta, Georgia, USA, in2004. From 1996 to 2000, he worked for LG Electronics Inc., Seoul, Korea. From 2004 to 2007, he was employed by the Samsung SDI R\&D Center, Yongin, Korea, as a Senior Engineer. From 2007 to 2008, he was with the Department of Electrical Engineering, Hanyang University, as a Research Professor. Since 2008, he has been an Associate Professor with the School of Electronic and Electrical Engineering, Hongik University, Seoul, Korea. His current research interests include variable speed drives and power conversion scheme for renewable energy sources and energy storage systems. 